Submitted to the Annals of Statistics

\title{
A CLASSIFICATION CRITERION FOR DEFINITIVE SCREENING DESIGNS
}

\author{
By Eric D. Schoen ${ }^{*, \dagger, \S}$, Pieter T. EendebaK ${ }^{\ddagger}$ And Peter Goos ${ }^{\dagger, \ddagger}$ \\ $K U$ Leuven $^{\dagger}$, University of Antwerp ${ }^{\ddagger}$ and $T N O^{\S}$
}

\begin{abstract}
A conference design is a rectangular matrix with orthogonal columns, one zero in each column, at most one zero in each row, and -1 s and +1 s elsewhere. A definitive screening design can be constructed by folding over a conference design and adding a row vector of zeroes. We prove that, for a given even number of rows, there is just one isomorphism class for conference designs with two or three columns. Next, we derive all isomorphism classes for conference designs with four columns. Based on our results, we propose a classification criterion for definitive screening designs based on projections into four factors and illustrate its potential by studying designs with 24 factors.
\end{abstract}

1. Introduction. Screening designs are helpful to study many controllable factors using a small number of experimental runs. The major part of the literature on screening designs focuses on two-level experiments; see Mee, Schoen and Edwards (2017) and Schoen, Vo-Thanh and Goos (2017) for recent reviews. The experimental results from a two-level screening design usually permit identification of substantial linear effects and, depending on the design, a few two-factor interactions. However, when all the factors are quantitative, it is of practical interest to investigate the presence of quadratic effects as well. For this purpose, Jones and Nachtsheim (2011) developed three-level designs using a number of runs that is one more than twice the number of factors studied. The designs are called definitive screening designs. Since their conception, a considerable effort has been invested into the further development of the definitive screening designs; references include Jones and Nachtsheim (2013), Georgiou, Stylianou and Aggarwal (2014), Nguyen and Pham (2016), Jones and Nachtsheim (2017) and Nachtsheim, Shen and Lin (2017). Recent applications have been described by Dougherty et al. (2015), Fidaleo et al. (2016) and Patil (2017).

The original definitive screening designs presented by Jones and Nachtsheim (2011) were based on a heuristic optimal design algorithm. For an odd

\footnotetext{
* Supported by the Research Foundation - Flanders FWO

MSC 2010 subject classifications: Primary 62K20; secondary 05B20, 94C30

Keywords and phrases: Conference Matrix, Isomorphism Class, $J_{4}$-characteristic, Projection 
number of factors and also for some even numbers of factors, these designs were not orthogonal for the linear effects. Xiao, Lin and Bai (2012) proposed constructing definitive screening designs that are orthogonal for the linear main effects using conference matrices. A conference matrix $\mathbf{C}$ of order $N$ is an $N \times N$ matrix with elements $c_{i j} \in\{-1,0,1\}$ such that $\mathbf{C}^{T} \mathbf{C}=(N-1) \mathbf{I}_{N}$, where $\mathbf{I}_{N}$ is an $N \times N$ identity matrix. The definition implies that there is one zero entry in every row and column of a conference matrix. A definitive screening design is constructed by folding over a conference matrix $\mathbf{C}$ and adding a row vector of zeroes. So, the structure of a definitive screening design is given by $\left[\mathbf{C}^{T},-\mathbf{C}^{T}, \mathbf{0}_{N}\right]^{T}$, where $\mathbf{0}_{N}$ is an $N$-dimensional column vector of zeroes. The orthogonality of a definitive screening design for the linear effects follows directly from the properties of a conference matrix.

Following Xiao, Lin and Bai (2012), the definitive screening designs studied in the current literature are all based on conference matrices. In this article, we adopt a more general approach, which is based on the notion of conference designs. A conference design $\mathbf{X}$ is an $N \times k$ matrix, with elements $x_{i j} \in\{-1,0,1\}$, columns $x_{1}, \ldots, x_{k}, k \leq N$ and at most one 0 in each row, such that $\mathbf{X}^{T} \mathbf{X}=(N-1) \mathbf{I}_{k}$. We refer to $N$ as the row size of the conference design. Clearly, a conference matrix is a special case of a conference design. In this paper, we use the name definitive screening design for any design constructed from an $N \times k$ conference design $\mathbf{X}$ by folding it over and adding a zero row. So the definitive screening designs we discuss have the form $\left[\mathbf{X}^{T},-\mathbf{X}^{T}, \mathbf{0}_{k}\right]^{T}$.

A justification of using small screening designs with many factors is the factor sparsity principle, which states that, generally, only a few factors are active. The end product of the data analysis then is a statistical model involving just a few linear effects, as well as some quadratic effects or twofactor interactions involving the active factors. It is therefore important to investigate the potential of screening designs for fitting models involving just a few factors. This can be done by studying projections of the screening designs onto smaller numbers of factors. Accordingly, projection properties form the basis of the generalized aberration criterion for classifying orthogonal arrays (Deng and Tang, 1999). The purpose of this paper is to develop a similar criterion for classifying definitive screening designs based on a study of their projections onto two, three and four factors. Since definitive screening designs are constructed by folding over conference designs, enumerating all their projections onto two, three and four factors is equivalent to enumerating all possible projections of conference designs onto two, three and four columns. Since enumerating conference designs is simpler, this is the route we take.

imsart-aos ver. 2014/10/16 file: AoS-DSDisomorphism_v2.tex date: May 7, 2018 
For a given row size $N$ and number of columns $k$, there can be many different conference designs. These can be grouped into isomorphism classes. The designs in one isomorphism class can be obtained from each other by a sequence of row permutations, column permutations, sign switches in columns or sign switches in rows. Unlike in orthogonal arrays, sign switches of rows in conference designs do result in isomorphic definitive screening designs, due to the fact that any such sign switch in the original conference design $\mathbf{X}$ causes an opposite sign switch in the folded-over conference design $-\mathbf{X}$. As a result, the definitive screening design is not affected by switching the signs in one or more rows of the original conference design. We therefore consider two conference designs that can be obtained from each other by switching the signs in entire rows as isomorphic.

Isomorphic designs have identical statistical properties, so that it suffices to study only one instance of each isomorphism class. We denote the set of isomorphism classes of conference designs with $N$ rows and $k$ columns by $C(N, k)$. To develop our classification criterion, the sets $C(N, k)$ for which $k$ equals 2,3 , or 4 are the most important. We focus on even values of $N$, because this is a necessary condition for conference designs to exist.

The rest of this paper is organized as follows. In Section 2, we show that all two-factor conference designs of a given row size $N$ are isomorphic. In Section 3, we derive a similar result for all three-factor conference designs of a given row size $N$. In Section 4, we derive all isomorphism classes of four-factor conference designs. Finally, based on the findings of the fourfactor conference designs, we propose a classification criterion for definitive screening designs in Section 5 and we illustrate its potential by studying all conference designs in $C(24,24)$.

2. Conference designs with two columns. For every even value of $N, C(N, 2)$ includes a single isomorphism class. To see this, consider any $N \times$ 2 conference design. By applying sign switches to the rows of the design and permuting the resulting rows, we can ensure that the first column, $x_{1}$, of the

design equals $\left[0, \mathbf{1}_{N-1}^{T}\right]^{T}$, where $\mathbf{1}_{z}$ denotes a $z$-dimensional column vector of ones, and that the second column, $x_{2}$, equals $\left[1,0, \mathbf{1}_{q}^{T},-\mathbf{1}_{q}^{T}\right]^{T}$, with $q=N / 2-$ 1. Because $C(N, 2)$ includes a single isomorphism class, projections onto two columns are not useful for classifying conference designs and definitive screening designs with more than two columns and a given value for $N$.

\section{Conference designs with three columns.}

3.1. Four design classes. At first sight, there are four possible ways to extend the two-column conference design with $x_{1}=\left[0, \mathbf{1}_{N-1}^{T}\right]^{T}$ and $x_{2}=$

imsart-aos ver. 2014/10/16 file: AoS-DSDisomorphism_v2.tex date: May 7, 2018 
$\left[1,0, \mathbf{1}_{q}^{T},-\mathbf{1}_{q}^{T}\right]^{T}$ from Section 2 by a third column, $x_{3}$. These extensions, labeled I-IV in Table 1, define four possible classes of three-column conference designs. For each of the classes, we set $x_{13}=1$. This can be done without loss of generality because, in the event $x_{13}$ would be -1 , we can always switch the signs of the elements in column $x_{3}$ and obtain a conference design that is isomorphic to the original one.

The $N$ rows of the four design classes in Table 1 are divided into three parts. The first part includes the row in which $x_{1}=0$ and $x_{2}=1$, and the row in which $x_{1}=1$ and $x_{2}=0$. The second part includes the $q=N / 2-1$ rows in with $x_{2}$ takes the value 1 , and the third part includes the $q=N / 2-1$ rows in with $x_{2}$ takes the value -1 .

The four classes in the table differ according to the value taken by $x_{3}$ at the second row (either 1 or -1 ) or according to the design part in which $x_{3}$ takes the value 0 (either part 2 or part 3 ). The number of times $x_{3}$ takes the value 1 in part 2 of the design is denoted by $q_{2}$, while the number of times it takes the value 1 in part 3 of the design is denoted by $q_{3}$.

3.2. Possible row sizes in the four classes. Since conference designs are orthogonal, the column $x_{3}$ has to be orthogonal to both the columns $x_{1}$ and $x_{2}$. This restricts the values of $N$ for which conference designs of the classes I, II, III and IV exist. For example, for class I, the columns $x_{1}$ and $x_{3}$ can only be orthogonal when

$$
1+q_{2}+q_{3}=q,
$$

while the columns $x_{2}$ and $x_{3}$ can only be orthogonal when

$$
q_{3}=q_{2}+1
$$

TABLE 1

Four classes of three-column conference designs

\begin{tabular}{llllclc}
\hline Part & $x_{1}$ & $x_{2}$ & $x_{3}(\mathrm{I})$ & $x_{3}(\mathrm{II})$ & $x_{3}(\mathrm{III})$ & $x_{3}(\mathrm{IV})$ \\
\hline 1 & 0 & 1 & 1 & 1 & 1 & 1 \\
& 1 & 0 & 1 & -1 & 1 & -1 \\
2 & $\mathbf{1}_{q}$ & $\mathbf{1}_{q}$ & 0 & 0 & $\mathbf{1}_{q_{2}}$ & $\mathbf{1}_{q_{2}}$ \\
& & & $\mathbf{1}_{q_{2}}$ & $\mathbf{1}_{q_{2}}$ & $-\mathbf{1}_{q-q_{2}}$ & $-\mathbf{1}_{q-q_{2}}$ \\
& & & $-\mathbf{1}_{q-1-q_{2}}$ & $-\mathbf{1}_{q-1-q_{2}}$ & & \\
3 & $\mathbf{1}_{q}$ & $-\mathbf{1}_{q}$ & $\mathbf{1}_{q_{3}}$ & $\mathbf{1}_{q_{3}}$ & 0 & 0 \\
& & & $-\mathbf{1}_{q-q_{3}}$ & $-\mathbf{1}_{q-q_{3}}$ & $\mathbf{1}_{q_{3}}$ & $\mathbf{1}_{q_{3}}$ \\
& & & & & $-\mathbf{1}_{q-1-q_{3}}$ & $-\mathbf{1}_{q-1-q_{3}}$ \\
\hline
\end{tabular}

imsart-aos ver. 2014/10/16 file: AoS-DSDisomorphism_v2.tex date: May 7, 2018 
TABLE 2

Two possible conference designs with 8 rows and 3 columns

\begin{tabular}{crrrrrr}
\hline Part & \multicolumn{3}{c}{ Class II } & \multicolumn{3}{c}{ Class III } \\
\hline 1 & 0 & 1 & 1 & 0 & 1 & 1 \\
& 1 & 0 & -1 & 1 & 0 & 1 \\
2 & 1 & 1 & 0 & 1 & 1 & 1 \\
& 1 & 1 & 1 & 1 & 1 & -1 \\
& 1 & 1 & -1 & 1 & 1 & -1 \\
& & & & & & \\
3 & 1 & -1 & 1 & 1 & -1 & 0 \\
& 1 & -1 & 1 & 1 & -1 & 1 \\
& 1 & -1 & -1 & 1 & -1 & -1 \\
\hline
\end{tabular}

Consequently, $q_{2}=q / 2-1$ and $q_{3}=q / 2$. As $q_{2}$ and $q_{3}$ have to be whole numbers, $q$ must be even. Since $N=2+2 q$, three-column conference designs of class I exist only when $N \equiv 2(\bmod 4)$.

Using a similar reasoning, we can show that conference designs of the classes II and III only exist when $N \equiv 0(\bmod 4)$, and that designs of the class IV only exist when $N \equiv 2(\bmod 4)$.

3.3. Isomorphism classes. When $N$ is a multiple of four, a three-column conference design can either belong to class II or to class III. Table 2 shows the designs from these two classes when $N=8$. The design of class III can be converted into the design of class II by swapping the second and third columns, sorting the rows so that the zeros are on the main diagonal, and sorting the remaining rows that do not contain zeros. Therefore, the designs of classes II and III are isomorphic for $N=8$. It is straightforward to show that this holds for all values of $N$ that are multiples of 4 . Thus, we have established the following result:

Lemma 1. When $N \equiv 0(\bmod 4), C(N, 3)$ includes a single isomorphism class.

When $N$ is an odd multiple of two, a three-column conference design can either belong to class I, or to class IV. Table 3 shows the designs for the two classes when $N=10$. The design of class IV can be converted into the design of class I in four steps. First, switch the signs in the second and third columns. Second, sort the rows so that the zero in the third column appears in the third row, while keeping the first two rows unchanged. Third, sort the remaining rows that do not contain zeros. Finally, switch the signs in the first row.

imsart-aos ver. 2014/10/16 file: AoS-DSDisomorphism_v2.tex date: May 7, 2018 
TABLE 3

Two possible conference designs with 10 rows and 3 columns

\begin{tabular}{rrrrrrr}
\hline Part & \multicolumn{3}{c}{ Class I } & \multicolumn{3}{c}{ Class IV } \\
\hline 1 & 0 & 1 & 1 & 0 & 1 & 1 \\
& 1 & 0 & 1 & 1 & 0 & -1 \\
2 & 1 & 1 & 0 & 1 & 1 & 1 \\
& 1 & 1 & 1 & 1 & 1 & 1 \\
& 1 & 1 & -1 & 1 & 1 & -1 \\
& 1 & 1 & -1 & 1 & 1 & -1 \\
3 & 1 & -1 & 1 & 1 & -1 & 0 \\
& 1 & -1 & 1 & 1 & -1 & 1 \\
& 1 & -1 & -1 & 1 & -1 & 1 \\
& 1 & -1 & -1 & 1 & -1 & -1 \\
\hline
\end{tabular}

Because we can convert the design of class IV into the design of class I using operations that preserve isomorphism, the designs of classes I and IV are isomorphic for $N=10$. It is straightforward to show that this holds for all even values of $N$ that are not multiples of 4 . Thus, we have established the following result:

Lemma 2. When $N \equiv 2(\bmod 4), C(N, 3)$ includes a single isomorphism class.

Combining both lemmas, we state our main result for the number of isomorphism classes in $C(N, 3)$ as follows:

Theorem 1. For any $N \equiv 0(\bmod 2), C(N, 3)$ includes a single isomorphism class.

Because $C(N, 3)$ includes a single isomorphism class for any given value of $N$, projections onto three columns are not useful for classifying conference designs and definitive screening designs with more than three columns.

\section{Conference designs with four columns.}

4.1. $J_{4}$-characteristics. The $J_{4}$-characteristic of a design in $C(N, 4)$ can be calculated in three steps. First, determine the elementwise products of the four columns. Second, sum these products. The resulting sum is the $j_{4}$-characteristic. Finally, the absolute value of the $j_{4}$-characteristic is the $J_{4}$-characteristic.

imsart-aos ver. 2014/10/16 file: AoS-DSDisomorphism_v2.tex date: May 7, 2018 
The correlation between two two-factor interaction contrast vectors involving all columns of a four-column conference design equals $j_{4} /(N-2)$. The sign of the $j_{4}$-characteristic changes if the signs of the elements in one or three of the four columns involved in its calculation are switched. The $j_{4}$-characteristic is not affected by any other operation that preserves isomorphism. So, its absolute value, the $J_{4}$-characteristic, is invariant to all operations that preserve isomorphism. For this reason, the $J_{4}$-characteristic is a key feature of a conference design with four columns.

Since a four-factor definitive screening design is constructed by folding over a four-column conference design, its $J_{4}$-characteristic is twice the $J_{4^{-}}$ characteristic of the conference design. The correlation between any two two-factor interaction contrast vectors involving all four factors is the same for the definitive screening design and for the original conference design.

4.2. Kernels of four-column conference designs. To enumerate non-isomorphic four-column conference designs, it is convenient to assume that both the first column and the first row start with a 0 and have 1s elsewhere, and that $x_{22}=x_{33}=x_{44}=0$. Define the kernel $\mathbf{K}$ of the conference design as

$$
\mathbf{K}=\left[\begin{array}{lll}
x_{22} & x_{23} & x_{24} \\
x_{32} & x_{33} & x_{34} \\
x_{42} & x_{43} & x_{44}
\end{array}\right]=\left[\begin{array}{ccc}
0 & x_{23} & x_{24} \\
x_{32} & 0 & x_{34} \\
x_{42} & x_{43} & 0
\end{array}\right]
$$

The kernel thus corresponds to the second, third and fourth column of rows 2, 3 and 4 of the conference design. The off-diagonal elements of $\mathbf{K}$ are either 1 or -1 . Delsarte, Goethals and Seidel (1971) studied the kernels of $N \times N$ conference matrices and found that they were symmetric or not depending on $N$. Here, we establish a similar result for kernels of $N \times 4$ conference designs.

Theorem 2. $\quad N \equiv 2(\bmod 4)$ if and only if $\mathbf{K}$ is symmetric. $N \equiv 0$ $(\bmod 4)$ if and only if $\mathbf{K}=-\mathbf{K}^{T}$.

Proof. To prove the sufficient part of Theorem 2, suppose first that $\mathbf{K}$ is symmetric. If $x_{32}=x_{23}=1$, the first three columns of any resulting four-column conference design form a three-column conference design that belongs to class I (see Table 3). If $x_{32}=x_{23}=-1$, the first three columns of any resulting four-column conference design form a three-column conference design that belongs to class IV (see again Table 3). Since class-I and classIV conference designs exist only when $N \equiv 2(\bmod 4)$, the row size $N$ of conference designs with symmetric kernels $\mathbf{K}$ must be such that $N \equiv 2$ $(\bmod 4)$.

imsart-aos ver. 2014/10/16 file: AoS-DSDisomorphism_v2.tex date: May 7, 2018 
Suppose further that $\mathbf{K}=-\mathbf{K}^{T}$ or antisymmetric. If $x_{32}=1$ and $x_{23}=$ -1 , the first three columns of any resulting four-column conference design form a three-column conference design that belongs to class II (see Table 2). If $x_{32}=-1$ and $x_{23}=1$, the first three columns of any resulting four-column conference design form a three-column conference design that belongs to class III (see again Table 2). Since the designs in classes II and III exist only when $N \equiv 0(\bmod 4)$, the row size $N$ of conference designs with opposite values for $x_{32}$ and $x_{23}$ must be a multiple of four.

To prove the necessary part of the theorem, we need to show that, when, $N \equiv 0(\bmod 4)$, the kernel must be antisymmetric, and that, when $N \equiv 2$ $(\bmod 4)$, the kernel must be symmetric. To this end, we use a proof by contradiction. First, suppose that $N \equiv 0(\bmod 4)$ and that $\mathbf{K}$ is not antisymmetric. In other words, suppose that $x_{32}=x_{23}$ or $x_{42}=x_{24}$ or $x_{43}=x_{34}$. By applying row and column permutations in the kernel, we can then always obtain a new kernel for which $x_{32}=x_{23}=1$ or $x_{32}=x_{23}=-1$. This produces a contradiction, because the first three columns of the resulting conference design would then possess the same structure as the designs in classes I or IV (see Table 3). However, designs in these classes exist only when $N \equiv 2(\bmod 4)$. So, if $N \equiv 0(\bmod 4)$, the elements of the pairs $\left(x_{32}, x_{23}\right)$, $\left(x_{42}, x_{24}\right)$ or $\left(x_{43}, x_{34}\right)$ must have opposite signs, in which case $\mathbf{K}=-\mathbf{K}^{T}$. Using the same kind of reasoning, we can show that, when $N \equiv 2(\bmod 4)$, the elements of the pairs $\left(x_{32}, x_{23}\right),\left(x_{42}, x_{24}\right)$ or $\left(x_{43}, x_{34}\right)$ must be equal, in which case $\mathbf{K}=\mathbf{K}^{T}$.

4.3. Four-column conference designs when the row size $N$ is a multiple of four. The rows of conference designs with $N \equiv 0(\bmod 4)$ can be arranged such that the first four rows contain the zeroes on the diagonal and have the structure shown in Table 4, where $p$ and $q$ are either 1 or -1 . We call this set of four rows the zero part of the design, and we call the remaining rows the nonzero part.

TABLE 4

The four rows containing zeroes in four-column conference designs whose row size is a multiple of 4

\begin{tabular}{rrrr}
\hline$x_{1}$ & $x_{2}$ & $x_{3}$ & $x_{4}$ \\
\hline 0 & 1 & 1 & 1 \\
1 & 0 & -1 & $-p$ \\
1 & 1 & 0 & $-q$ \\
1 & $p$ & $q$ & 0 \\
\hline
\end{tabular}

imsart-aos ver. 2014/10/16 file: AoS-DSDisomorphism_v2.tex date: May 7, 2018 
The zero part can include one of four different pairs $(p, q)$. Three of these pairs, $(1,1),(1,-1)$ and $(-1,-1)$, give rise to isomorphic conference designs. This can be verified by permuting the rows and columns of the resulting conference designs. For each of these cases, four of the six pairs of columns in the zero part in Table 4 have inner products of \pm 2 , and the remaining two pairs have inner products of 0 . Conference designs constructed using the fourth pair, $(-1,1)$, are not isomorphic to those constructed with one of the other three pairs, because all pairs of columns in the zero part in Table 4 have inner products of 0 when using that fourth pair. We call the series of conference designs constructed using one of the pairs $(1,1),(1,-1)$ and $(-1,-1)$ series $\mathrm{A}$, and the series of conference designs constructed using the pair $(-1,1)$ series B. In the remainder of this section, we assume, without loss of generality, that the pair $(1,1)$ is chosen to construct the conference designs in series A.

In the event $(p, q)=(1,1)$, the zero part of the conference design is given by the first four rows in the left panel of Table 5 . The six inner products of all pairs of columns in that design part equal 2 (columns $x_{1}$ and $x_{2}$ ), 0 (columns $x_{1}$ and $x_{3}$ ), -2 (columns $x_{1}$ and $x_{4}$ ), 2 (columns $x_{2}$ and $x_{3}$ ), 0 (columns $x_{2}$ and $\left.x_{4}\right)$ and 2 (columns $x_{3}$ and $x_{4}$ ). This implies that the $4 \times 4$ matrix in Table 4 is not orthogonal when $(p, q)=(1,1)$, and that it is not a conference design. However, it can be used to build a conference design with $N=4(g+1)$ rows by adding $4 g$ rows of +1 s and -1 s for which the inner products of the column pairs equal $-2,0,2,-2,0$ and -2 , respectively. These $4 g$ added rows form the nonzero part of the conference design, and they ensure that the inner products of all pairs of columns, calculated across the entire design, equal zero, so that all columns are orthogonal. In the Appendix, we prove that any conference design in series A must contain the set of four rows from the nonzero part of the design in the left panel of Table 5 if the zero part is identical to that in the top left part of Table 5. The design shown in Table 5 therefore represents the smallest possible conference design in series A, and any larger four-column conference design is an extension of it involving additional rows, or is isomorphic to such an extension. The $J_{4^{-}}$ characteristic of the smallest series-A conference design in the left panel of Table 5, involving eight rows, equals 0 .

After identifying the smallest conference design in series $\mathrm{A}$, the next challenge is to study the designs with larger row sizes $N$. To derive all isomorphism classes in series A for a given row size $N$, we assume, without loss of generality, that the first eight rows are those shown in Table 5's left panel. Conference designs of series A with $N \geq 12$ can thus be constructed by adding a set of $z=4(g-1)$ extra rows to the initial eight. Without loss 
TABLE 5

Smallest four-column conference designs in series $A$ and series $B$

\begin{tabular}{rrrrrrrr}
\multicolumn{3}{c}{ Series A } & \multicolumn{5}{c}{ Series B } \\
\hline$x_{1}$ & $x_{2}$ & $x_{3}$ & $x_{4}$ & $x_{1}$ & $x_{2}$ & $x_{3}$ & $x_{4}$ \\
\hline 0 & 1 & 1 & 1 & 0 & 1 & 1 & 1 \\
1 & 0 & -1 & -1 & 1 & 0 & -1 & 1 \\
1 & 1 & 0 & -1 & 1 & 1 & 0 & -1 \\
1 & 1 & 1 & 0 & 1 & -1 & 1 & 0 \\
& & & & & & & \\
1 & 1 & -1 & 1 & & & & \\
1 & -1 & 1 & 1 & & & & \\
1 & -1 & 1 & -1 & & & & \\
1 & -1 & -1 & 1 & & & & \\
\hline
\end{tabular}

of generality, the first column in the extra set can be taken to be $\mathbf{1}_{z}$. To preserve the orthogonality of the columns in the complete $N$-row conference design, the columns 2, 3 and 4 of the $z$ additional rows must then form a two-level orthogonal array with $z$ rows, three columns and levels -1 and +1 .

Because the design in Table 5 has a $J_{4}$-characteristic of 0 and the $z$ entries of the first column of the extra rows are all 1 , the $J_{4}$-characteristic of the complete design is determined entirely by the aliasing structure of the orthogonal array used. More specifically, the $J_{4}$-characteristic of the complete design is equal to the $J_{3}$-characteristic of the orthogonal array, which is calculated by taking the elementwise products of its columns, summing these products to obtain the $j_{3}$-characteristic, and taking the absolute value of that $j_{3}$-characteristic. Deng and Tang (1999, proposition 2ii) show that any $z$-row orthogonal array with three columns and a certain $J_{3}$-characteristic can be constructed by concatenating $\left[z-J_{3}\right] / 8$ copies of a full factorial $2^{3}$ design and $J_{3} / 4$ copies of one of its half fractions. Denoting the three columns by $X_{2}, X_{3}$ and $X_{4}$, each of the two possible half fractions includes all four combinations of the \pm 1 entries for $X_{2}$ and $X_{3}$. For one of the fractions, the entries for $X_{4}$ are calculated as $X_{4}=X_{2} X_{3}$, while, for the other fraction, the entries for $X_{4}$ are calculated as $X_{4}=-X_{2} X_{3}$. However, if the latter option is chosen, applying sign switches to the columns $2-4$, applying a sign switch to the first row, and permuting columns and rows, the corresponding conference design can be converted into the conference design based on the former option. Therefore, the two ways of defining the half fraction lead to isomorphic conference designs, so that, without loss of generality, we consider only the half fraction for which $X_{4}=X_{2} X_{3}$.

Deng and Tang (1999) also point out that the $J_{3}$-characteristic of any $z$-row orthogonal array involving three two-level factors is a multiple of 4 , 
TABLE 6

Possible $J_{4}$-characteristics and isomorphism classes for conference designs whose row size $N$ is a multiple of 4

\begin{tabular}{|c|c|c|c|c|c|c|}
\hline Row Size & \multicolumn{3}{|c|}{ Series A } & \multicolumn{3}{|c|}{ Series B } \\
\hline 4 & - & & & 0 & & \\
\hline 8 & 0 & & & 4 & & \\
\hline 12 & 4 & & & 8 & 0 & \\
\hline 16 & 8 & 0 & & 12 & 4 & \\
\hline 20 & 12 & 4 & & 16 & 8 & 0 \\
\hline 24 & 16 & 80 & 0 & 20 & 12 & 4 \\
\hline$N$ & \multicolumn{2}{|c|}{$\lambda=1, \ldots,\lfloor N / 8\rfloor$} & & \multicolumn{3}{|c|}{$(N-4)-8 \lambda$} \\
\hline
\end{tabular}

of the form $z-8 h$, where $h \leq z / 8$ is a nonnegative integer. Therefore, the $J_{3}$-characteristic is a multiple of 8 if $z \equiv 0(\bmod 8)$ and an odd multiple of 4 if $z \equiv 4(\bmod 8)$. Because the $J_{4}$-characteristic of any entire fourcolumn conference design in series $\mathrm{A}$ is given by the $J_{3}$-characteristic of the orthogonal array used in the $z$ extra rows, any conference design in series A will have a $J_{4}$-characteristic of the form $z-8 h$, where $z=N-8$. Equivalently, $J_{4}=N-8 \lambda$, where $\lambda \leq N / 8$ is a strictly positive integer.

In the event $(p, q)=(-1,1)$, the $4 \times 4$ matrix in Table 4 is a conference design with a $J_{4}$-characteristic of 0 . So, the smallest conference design in series B has $N=4$. That design is shown in the right panel of Table 5 . The enumeration of all isomorphism classes of series B is otherwise similar to that for series A. Adding $z=N-4$ rows to the smallest conference design of this series results in $J_{4}$-characteristics of $z-8 h=N-4-8 \lambda$, where $\lambda \leq(N-4) / 8$ is a nonnegative integer.

Table 6 shows the possible values of the $J_{4}$-characteristics of the conference designs in series $\mathrm{A}$ and series $\mathrm{B}$ for $4,8, \ldots, 24$ rows and for a general $N$ value that is a multiple of four. Each isomorphism class is uniquely determined by its $J_{4}$-characteristic. The numbers of isomorphism classes in series $\mathrm{A}$ and series $\mathrm{B}$ equal $\lfloor N / 8\rfloor$ and $\lceil N / 8\rceil$, respectively. So, the total number of isomorphism classes for a given value of $N$ is $N / 4$. We summarize the results obtained in this section by the following theorem:

TheOREM 3. For row sizes $N \equiv 0(\bmod 4)$, the following properties hold for the designs in $C(N, 4)$ :

1. The number of isomorphism classes equals $N / 4$.

2. Each isomorphism class is uniquely determined by the value of its $J_{4}$ characteristic. 
3. The possible values of the $J_{4}$-characteristic are $N-4 \lambda$, for $\lambda=1, \ldots, N / 4$.

4.4. Four-column conference designs when the row size $N$ is an odd multiple of two. Without loss of generality, we assume that the first four rows of conference designs with $N \equiv 2(\bmod 4)$ correspond to those in Table 7 , where $p$ and $q$ are either 1 or -1 . As before, we refer to these four rows as the zero part of the conference designs, and to the rows of \pm 1 s we add as the nonzero part. There are four possible pairs $(p, q)$. However, by permuting rows and columns, it can be shown that the designs for $(p, q)=(1,-1)$ and $(p, q)=(-1,1)$ are isomorphic. In addition, by applying sign switches in the last three columns and permuting the resulting rows and columns, it can be shown that the design for $(p, q)=(-1,-1)$ is isomorphic to those for $(p, q)=(1,-1)$ and $(p, q)=(-1,1)$. For these three cases, the inner products of the columns equal 0 for four of the column pairs and \pm 2 for two of the pairs. The design for $(p, q)=(1,1)$ is not isomorphic to the other three designs, because all inner products equal 2 . We call the conference design series for which $(p, q)$ is $(1,1)$ series $\mathrm{C}$, and the series for which $(p, q)$ is $(1,-1)$, $(-1,1)$ or $(-1,-1)$ series $\mathrm{D}$. To construct designs that are representatives for the isomorphism classes in series $\mathrm{D}$, we use the pair $(p, q)=(1,-1)$ in the remainder of this section. The first four rows in the left and right panels of Table 8 show the zero parts for the representatives in series $\mathrm{C}$ and D.

We start by studying the isomorphism classes in series C. As the inner product of any pair of columns in Table 7 equals 2 when $(p, q)=(1,1)$, conference designs in this series require $4 g+2$ additional rows of 1 s and $-1 \mathrm{~s}$, for which the inner product of any pair of columns is -2 . In the online Appendix, we prove that any conference design of series $\mathrm{C}$ must contain the six rows of the nonzero part of the design in the left panel of Table 8 . Therefore, $g$ should be a strictly positive integer for series C, and the 10row design shown in the table is the smallest possible conference design in series C. Its $j_{4}$-characteristic equals 6 .

To construct conference designs from series D starting from Table 7, we

TABLE 7

The four rows containing zeroes in four-column conference designs whose row size is an odd multiple of two

\begin{tabular}{rrrr}
\hline$x_{1}$ & $x_{2}$ & $x_{3}$ & $x_{4}$ \\
\hline 0 & 1 & 1 & 1 \\
1 & 0 & 1 & $p$ \\
1 & 1 & 0 & $q$ \\
1 & $p$ & $q$ & 0 \\
\hline
\end{tabular}

imsart-aos ver. 2014/10/16 file: AoS-DSDisomorphism_v2.tex date: May 7, 2018 
TABLE 8

Smallest four-column conference designs within series $C$ and series $D$

\begin{tabular}{rrrrrrrr}
\hline & \multicolumn{3}{c}{ Series C } & \multicolumn{5}{c}{ Series D } \\
$x_{1}$ & $x_{2}$ & $x_{3}$ & $x_{4}$ & $x_{1}$ & $x_{2}$ & $x_{3}$ & $x_{4}$ \\
\hline 0 & 1 & 1 & 1 & 0 & 1 & 1 & 1 \\
1 & 0 & 1 & 1 & 1 & 0 & 1 & 1 \\
1 & 1 & 0 & 1 & 1 & 1 & 0 & -1 \\
1 & 1 & 1 & 0 & 1 & 1 & -1 & 0 \\
& & & & & & & \\
1 & 1 & -1 & -1 & 1 & -1 & 1 & -1 \\
1 & 1 & -1 & -1 & 1 & -1 & -1 & 1 \\
1 & -1 & 1 & -1 & & & & \\
1 & -1 & 1 & -1 & & & & \\
1 & -1 & -1 & 1 & & & & \\
1 & -1 & -1 & 1 & & & & \\
\hline
\end{tabular}

need to add a matrix involving $4 g+2$ rows of 1 s and -1 s for which the inner product of the columns $x_{1}$ and $x_{2}$ is 2 , that of the columns $x_{3}$ and $x_{4}$ is 2 as well, and the inner products of the other column pairs are 0 . In the Appendix, we prove that any conference design of series D must contain the two rows of the nonzero part of the design in the right panel of Table 8 if the zero part is identical to that in the table's right panel. Therefore, $g$ can be any nonnegative integer for series $\mathrm{D}$, and the 6-row design shown in the table is the smallest possible conference design in series D. Its $j_{4^{-}}$ characteristic equals 2 .

After identifying the smallest conference designs in series $\mathrm{C}$ and $\mathrm{D}$, the next challenge is to study the designs with larger row sizes $N$, where $N$ is an odd multiple of two. We assume, without loss of generality, that the first ten rows of any $N \times 4$ conference design of series $\mathrm{C}$ and the first six rows of any $N \times 4$ conference design of series D are those shown in Table 8 . To obtain conference designs with larger row sizes, we have to add sets of $z$ rows of $1 \mathrm{~s}$ and $-1 \mathrm{~s}$ to these two designs. Without loss of generality, the first column in the extra sets can again be taken to be $\mathbf{1}_{z}$. To preserve the orthogonality of all columns in the complete conference design, the columns 2, 3 and 4 of the $z$ extra rows must then form a two-level orthogonal array with $z$ rows. As all entries of column 1 equal 1 , the $j_{4}$-characteristic of the $z$ added rows, which form the nonzero part of the design, is equal to the $j_{3}$-characteristic of the orthogonal array with $z$ rows used for columns 2,3 , and 4 . The $j_{4}$-characteristic of the complete conference design now is the sum of the $j_{4}$-characteristic of the zero part of the design and that of the nonzero part, while the $J_{4}$-characteristic is its absolute value. 
TABLE 9

Possible $J_{4}$-characteristics and isomorphism classes for conference designs whose row size $N$ is an odd multiple of 2

\begin{tabular}{|c|c|c|c|c|c|c|c|}
\hline Row Size & \multicolumn{2}{|l|}{ Series C } & \multicolumn{5}{|c|}{ Series D } \\
\hline 6 & - & & 2 & & & & \\
\hline 10 & 6 & & 6 & 2 & & & \\
\hline 14 & 10 & & 10 & 2 & 6 & & \\
\hline 18 & 14 & 2 & 14 & 6 & 2 & 10 & \\
\hline 22 & $18 \quad 10$ & 26 & 18 & 10 & 2 & 6 & 14 \\
\hline$N$ & $\begin{array}{l}\mid N-4(2 \\
\lambda=0, \ldots\end{array}$ & $\begin{array}{l}\lambda+1) \mid \\
,(N-10) / 4\end{array}$ & & $\begin{array}{l}-4(2 \\
0, \ldots\end{array}$ &,$(1$ & $\begin{array}{l}\mid \\
-6)\end{array}$ & \\
\hline
\end{tabular}

Unlike the smallest conference designs in series A or series B for rows sizes $N$ that are multiples of four, those in series $\mathrm{C}$ or series $\mathrm{D}$ have nonzero $j_{4}$-characteristics. More specifically, their $j_{4}$-characteristics equal 6 and 2 , respectively. Therefore, utilizing a $z$-row orthogonal array with a $j_{3}$-characteristic of $z-8 h$ for the columns $2-4$ when adding $z$ rows to the smallest designs in series $\mathrm{C}$ and series $\mathrm{D}$ results in a $j_{4}$-characteristic $6+z-8 h$ or $2+z-8 h$, respectively, for the entire design. Using the mirror image of the orthogonal array, which has a $j_{3}$-characteristic of $8 h-z$, would result in $j_{4}$-characteristics of $6-z+8 h$ and $2-z+8 h$, respectively. Therefore, unlike for the conference designs in series A and series B, using the original orthogonal array or its mirror image results in different $J_{4}$-characteristics, unless the $z$-row orthogonal array used has a $J_{3}$-characteristic of zero. Any $z$-row orthogonal array with a nonzero $J_{3}$-characteristic therefore gives rise to two non-isomorphic conference designs in series $\mathrm{C}$ and in series $\mathrm{D}$.

For an $N$-row conference design in series $\mathrm{C}, z=N-10$. Therefore, its $j_{4}$-characteristic equals $N-4-8 h$ or $-N+16+8 h$, where $h \leq(N-10) / 8$. Equivalently, it is of the form $N-4(2 \lambda+1)$, where $\lambda \leq(N-10) / 4$ is a nonnegative integer. For an $N$-row conference design in series $\mathrm{D}, z=N-6$. Therefore, its $j_{4}$-characteristic equals $N-4-8 h$ or $-N+8+8 h$, where $h \leq(N-6) / 8$. So, it is also of the form $N-4(2 \lambda+1)$, but, for series $\mathrm{D}, \lambda$ is a nonnegative integer of at most $(N-6) / 4$.

Table 9 shows the possible $J_{4}$-characteristics of the conference designs in series $\mathrm{C}$ and series $\mathrm{D}$ for $6,10, \ldots, 22$ rows and for a general $N$ value that is an odd multiple of two. The numbers of isomorphism classes in series $\mathrm{C}$ and series D equal $\lfloor(N-4) / 4\rfloor$ and $\lceil(N-4) / 4\rceil$, respectively. So, the total number of isomorphism classes equals $(N-4) / 2$ when $N$ is an odd multiple of two. The $J_{4}$-characteristics in both series are odd multiples of two. Unlike when 
$N$ is a multiple of four, the isomorphism classes in series $\mathrm{C}$ and $\mathrm{D}$ for a given row size $N$ are not uniquely identified by their $J_{4}$-characteristic, except for the class whose $J_{4}$-characteristic equals $|8-N|$. When the $J_{4}$-characteristic differs from $|8-N|$, the zero part of the matrix can be used to determine whether the design belongs to series $\mathrm{C}$ or $\mathrm{D}$ by checking the inner products of its columns. The design belongs to series $\mathrm{C}$ if the inner products are all nonzero, and to series D otherwise. We summarize the results as follows:

TheOREM 4. For row sizes $N \equiv 2(\bmod 4)$, the following properties hold for the designs in $C(N, 4)$ :

1. The number of isomorphism classes equals $(N-4) / 2$.

2. The possible values of the $J_{4}$-characteristic are $|N-4(2 \lambda+1)|$ for $\lambda=0, \ldots,(N-6) / 4$.

3. The $J_{4}$-characteristic of $|N-8|$ uniquely determines one of the isomorphism classes for a given $N$ value.

4. The remaining isomorphism classes can be divided into pairs with unique $J_{4}$-characteristics.

5. A generalized aberration criterion for definitive screening designs. By studying the information matrix of a response surface model, including the intercept and all linear effects, quadratic effects and two-factor interactions, it is easy to establish the following properties of model matrix columns for any definitive screening design: (1) linear effects are orthogonal to each other, (2) linear effects are orthogonal to quadratic effects, (3) linear effects are orthogonal to two-factor interactions, (4) the inner product of any pair of quadratic effect columns equals $2 N-4,(5)$ the inner product of any quadratic effect column involving a given factor with a two-factor interaction column involving that factor equals 0 , and (6) the inner product of any quadratic effect column involving a factor with a two-factor interaction column not involving that factor equals \pm 2 ; see Jones and Nachtsheim (2011).

For a given run size $2 N+1$ and a given number of factors $k$, there may be several non-isomorphic definitive screening designs. When considering the properties of the columns of the response surface model's model matrix, the inner products of pairs of two-factor interaction columns are the only variable features in a definitive screening design of a given run size. This suggests that we should rank the definitive screening designs according to the extent to which two-factor interactions are aliased. We can do this by considering the $J_{4}$-characteristics of all four-factor projections of the definitive screening designs. As definitive screening designs and their projections

imsart-aos ver. 2014/10/16 file: AoS-DSDisomorphism_v2.tex date: May 7, 2018 
are folded-over conference designs, the possible $J_{4}$-characteristics of fourfactor projections of definitive screening designs are readily obtained from Theorems 3 and 4 . More specifically, the $J_{4}$-characteristic of a four-factor definitive screening design is twice the $J_{4}$-characteristic of the corresponding conference design. Likewise, the $J_{4}$-characteristic of any possible four-factor projection of a definitive screening design is twice the $J_{4}$-characteristic of the corresponding four-column projection of the corresponding conference design. Theorems 3 and 4 therefore have the following corollary:

COROllary 1. For any four-factor definitive screening design involving $2 N+1$ runs:

1. If $N \equiv 0(\bmod 4)$, the $N / 4$ possible values of the $J_{4}$-characteristics are $2 N-8,2 N-16, \ldots, 0$.

2. If $N \equiv 2(\bmod 4)$, the $(N-4) / 2$ possible values of the $J_{4}$-characteristics are $2 N-8,2 N-16, \ldots, 4$.

When a four-factor definitive screening design has to be selected for an experiment, it is best to pick one that minimizes the $J_{4}$-characteristic. This is because the absolute correlation between each of the three pairs of two-factor interaction contrast vectors involving all four factors equals $J_{4} /(2 N-4)$. Small correlations between the contrast vectors result in small correlations for the estimators in any model involving two two-factor interactions and a smaller bias in any model involving only one of the two-factor interactions.

When a definitive screening design with more than four factors has to be selected, we suggest determining the frequency vector $F_{4}$ of the $J_{4^{-}}$ characteristics of $2 N-8 \lambda$ for $\lambda=1, \ldots, N / 4$ when $N$ is a multiple of 4 , or $\lambda=1, \ldots,(N-2) / 4$ when $N$ is an odd multiple of 2 . The definitive screening designs under consideration are then ordered based on that vector. More specifically, we sort the designs in ascending order of the $F_{4}$ vector's first entry. Designs with the same first entry are sorted in ascending order of the second entry. The process continues until a unique order has been established or all entries have been considered. We define the generalized aberration of a definitive screening design as its rank after the sorting procedure. A design with rank 1 thus has a minimum generalized aberration.

The generalized aberration criterion for orthogonal two-level designs of Deng and Tang (2002) is based on the confounding frequency vector $\left(F_{3}, F_{4}\right.$, $\ldots, F_{k}$ ), where $F_{i}$ denotes the frequency vector of the nonzero $J_{i}$-characteristics and $k$ is the number of factors or columns in the design. We base our generalized aberration criterion for definitive screening designs on the $F_{4}$ vector only for the following reasons. First, definitive screening designs are

imsart-aos ver. 2014/10/16 file: AoS-DSDisomorphism_v2.tex date: May 7, 2018 
TABLE 10

$F_{4}$ vectors for all 49-run definitive screening designs involving 24 factors

\begin{tabular}{lrrrrrr}
\hline Design & \multicolumn{5}{c}{$F_{4}(40,32,24,16,8,0)$} \\
\hline 1 & 0 & 0 & 0 & 3036 & 3036 & 4554 \\
2 & 18 & 12 & 114 & 2652 & 2904 & 4926 \\
3 & 24 & 12 & 120 & 2604 & 2892 & 4974 \\
4 & 24 & 16 & 120 & 2588 & 2892 & 4986 \\
5 & 24 & 21 & 150 & 2508 & 2862 & 5061 \\
6 & 24 & 24 & 192 & 2412 & 2820 & 5154 \\
7 & 30 & 24 & 150 & 2460 & 2856 & 5106 \\
8 & 36 & 20 & 180 & 2380 & 2820 & 5190 \\
9 & 66 & 0 & 330 & 1980 & 2640 & 5610 \\
\hline
\end{tabular}

fold-over designs, so that any $J_{i}$-characteristic for an odd value of $i$ is zero. This is due to the fact that the $J_{i}$-characteristic of a conference design's mirror image is the negative of the $J_{i}$-characteristic of the original conference design when $i$ is odd. As a result, $F_{i}$ vectors are zero vectors when $i$ is odd, for any definitive screening design, and therefore do not help to distinguish alternative design screening design options. Second, the $F_{i}$ vectors for even values of $i$ larger than 4 do not contain information that is relevant to experimenters. As a matter of fact, these vectors quantify the aliasing of contrast vectors corresponding to third-order and higher-order interaction contrast vectors. Generally, such interactions are unimportant and most experimenters assume they are negligible.

To illustrate the usefulness of our generalized aberration criterion for definitive screening designs, we studied cases with 24 factors in 49 runs and 82 factors in 165 runs. For the first of these cases, we generated representatives of all isomorphism classes of conference matrices of order 24 with a dedicated computer program. This resulted in 9 different conference matrices, which confirms the number of isomorphism classes established by Greig, Haanpää and Kaski (2006). We determined the $F_{4}$ vectors of the corresponding definitive screening designs and ordered the designs according to their generalized aberration. The $F_{4}$ vectors are shown in Table 10.

Since the possible nonzero $J_{4}$-characteristics for conference designs with $N=24$ equal $20,16,12,8$ and 4 (see Table 6 ), the possible nonzero $J_{4^{-}}$ characteristics for the corresponding definitive screening designs are 40, $32,24,16$ and 8 . The best of the nine non-isomorphic designs has $J_{4^{-}}$ characteristics of at most 16 and a maximum absolute correlation of 4/11 between pairs of two-factor interaction contrast vectors. In total, there are $3 \times 3036=9108$ pairs of interactions with this absolute correlation. For the 
TABLE 11

$F_{4}$ vectors for the 165-run definitive screening designs involving 82 factors based on the 26 conference designs of Hurkens and Seidel (1985). For all the designs, $\max \left(J_{4}\right) \leq 100$ and $F_{4}(4)$ equals 1749060 minus the sum of the frequencies stated in the table

\begin{tabular}{rrrrrrrrrrrrr}
\hline Design & \multicolumn{1}{c|c}{$F_{4}(100,92,84,76,68,60,52,44,36,28,20,12)$} \\
\hline 2 & 0 & 0 & 0 & 0 & 0 & 0 & 0 & 0 & 22140 & 398520 & 531360 & 0 \\
4 & 0 & 0 & 0 & 0 & 0 & 2700 & 0 & 55080 & 139320 & 116640 & 252720 & 453600 \\
13 & 0 & 0 & 0 & 720 & 320 & 3100 & 10080 & 32200 & 83800 & 173600 & 340080 & 481360 \\
23 & 0 & 38 & 4 & 196 & 436 & 3406 & 8584 & 30800 & 84790 & 178684 & 345876 & 484092 \\
24 & 0 & 38 & 4 & 196 & 436 & 3406 & 8584 & 30800 & 84790 & 178684 & 345876 & 484092 \\
21 & 0 & 54 & 108 & 0 & 108 & 7290 & 3024 & 36612 & 92448 & 163134 & 330912 & 480384 \\
22 & 0 & 72 & 18 & 216 & 360 & 4092 & 10728 & 31770 & 82332 & 172872 & 340452 & 479856 \\
7 & 0 & 156 & 0 & 128 & 272 & 3792 & 7360 & 31072 & 84104 & 181216 & 349984 & 475648 \\
8 & 0 & 156 & 0 & 128 & 272 & 3792 & 7360 & 31072 & 84104 & 181216 & 349984 & 475648 \\
15 & 0 & 244 & 0 & 0 & 480 & 4512 & 7168 & 31552 & 84672 & 173424 & 352064 & 479616 \\
11 & 0 & 252 & 0 & 288 & 288 & 4608 & 5184 & 25920 & 87768 & 185616 & 364608 & 454752 \\
12 & 0 & 252 & 0 & 288 & 288 & 4608 & 5184 & 25920 & 87768 & 185616 & 364608 & 454752 \\
3 & 0 & 486 & 0 & 0 & 0 & 8046 & 3888 & 30780 & 92664 & 173502 & 331452 & 443880 \\
1 & 0 & 4212 & 0 & 0 & 0 & 13824 & 5184 & 36288 & 104544 & 53136 & 336960 & 513216 \\
25 & 2 & 12 & 15 & 186 & 439 & 3364 & 8441 & 31468 & 85416 & 178286 & 343555 & 482639 \\
26 & 2 & 12 & 15 & 186 & 439 & 3364 & 8441 & 31468 & 85416 & 178286 & 343555 & 482639 \\
14 & 8 & 6 & 24 & 144 & 816 & 3182 & 8624 & 31556 & 83368 & 181894 & 340060 & 479640 \\
16 & 18 & 0 & 0 & 594 & 540 & 4320 & 8406 & 28314 & 85005 & 182907 & 338202 & 474732 \\
17 & 18 & 0 & 0 & 594 & 540 & 4320 & 8406 & 28314 & 85005 & 182907 & 338202 & 474732 \\
18 & 36 & 234 & 0 & 216 & 918 & 4698 & 7740 & 30096 & 86670 & 174672 & 337896 & 471636 \\
19 & 36 & 234 & 0 & 216 & 918 & 4698 & 7740 & 30096 & 86670 & 174672 & 337896 & 471636 \\
5 & 52 & 108 & 0 & 288 & 700 & 4608 & 8856 & 28368 & 84212 & 178392 & 339592 & 485488 \\
6 & 52 & 108 & 0 & 288 & 700 & 4608 & 8856 & 28368 & 84212 & 178392 & 339592 & 485488 \\
20 & 72 & 90 & 72 & 144 & 648 & 4398 & 9360 & 33192 & 79020 & 173358 & 348696 & 467256 \\
9 & 396 & 756 & 0 & 2376 & 2916 & 7056 & 9648 & 23832 & 81972 & 158904 & 296784 & 492768 \\
10 & 396 & 756 & 0 & 2376 & 2916 & 7056 & 9648 & 23832 & 81972 & 158904 & 296784 & 492768 \\
\hline
\end{tabular}

other eight designs, the $J_{4}$-characteristics can be as large as 40 , corresponding to an absolute correlation of 10/11 and indicating almost complete aliasing between certain pairs of two-factor interactions. Therefore, we strongly recommend to employ the first of the non-isomorphic 24-factor definitive screening designs in Table 10. The corresponding conference design is given explicitly in Appendix B.

Hurkens and Seidel (1985) specified how to construct a series of 26 nonisomorphic conference matrices of order 82 based on sets of mutually orthogonal Latin squares. Based on these matrices, we constructed definitive screening designs with 165 runs and 82 factors. We determined the $F_{4}$ vectors of these designs and ordered the designs according to their generalized 
aberration. The results are shown in Table 11; the design labels correspond to those in Hurkens and Seidel (1985). A Matlab program that outputs the conference matrices as well as the $F_{4}$ vectors is provided as supplemental material to this paper (Schoen, 2018).

Since the possible nonzero $J_{4}$-characteristics for conference designs with $N=82$ equal $78,74, \ldots, 2$ (see Table 9 ), the possible $J_{4}$-characteristics for the corresponding definitive screening designs are $156,148, \ldots, 4$. There are thus no non-zero $J_{4}$-characteristics. This implies that $F_{4}(4)=\left(\begin{array}{c}82 \\ 4\end{array}\right)-$ $\Sigma F_{4}\left(J_{4}>4\right)=1749060-\Sigma F_{4}\left(J_{4}>4\right)$, where the sum adds all frequencies of $J_{4}$-characteristics larger than 4 .

It turns out that none of the 26 definitive screening designs have $J_{4^{-}}$ characteristics larger than 100. The best of the 26 non-isomorphic designs has $J_{4}$-characteristics of at most 36 and a maximum absolute correlation of $9 / 40=0.225$ between pairs of two-factor interaction contrast vectors. In total, there are $3 \times 22140=66420$ pairs of interactions with this absolute correlation. The second best and third best designs have pairs of interactions with absolute correlations up to 0.375 and 0.475 , respectively, while the remaining designs have correlations as large as 0.575 (11 cases) or 0.675 (12 cases). Therefore, we strongly recommend to employ the first of the non-isomorphic 82-factor definitive screening designs in Table 11.

\section{APPENDIX A: COMPULSORY ROWS IN $N \times 4$ CONFERENCE DESIGNS}

The purpose of this Appendix is to prove that certain rows must be present in the nonzero parts of $N \times 4$ conference designs. The general shape of these parts is shown in Table 12. The first column can be taken to contain only +1 elements. It is convenient to use the symbol $k$ for $m-1$. So, if $N=4 m$, the number of rows in the nonzero part equals $z=4 k$. In case $N=4 m+2$, the number of rows in the nonzero part is $z=4 k+2$. The symbols $a, b, c, d, e, f, p, q, r, s, u, v$ and $w$ define nonnegative numbers of rows for the various level combinations of the columns $x_{2}, x_{3}$ and $x_{4}$ in the nonzero part of a conference design. For example, there are $a$ rows with a +1 for $x_{2}$, there are $c$ rows with $\mathrm{a}+1$ for $x_{2}$ and a +1 for $x_{3}$, and so on.

A.1. Series A. For the initial four rows of an $N \times 4$ conference design in series A, the six inner products of the column pairs in Table 5 equal 2 (columns $x_{1}$ and $x_{2}$ ), 0 (columns $x_{1}$ and $x_{3}$ ), -2 (columns $x_{1}$ and $x_{4}$ ), 2 (columns $x_{2}$ and $x_{3}$ ), 0 (columns $x_{2}$ and $x_{4}$ ) and 2 (columns $x_{3}$ and $x_{4}$ ), which implies that the $4 \times 4$ matrix in Table 5 is not orthogonal. Therefore, it is not a conference design. However, it can be turned into a conference

imsart-aos ver. 2014/10/16 file: AoS-DSDisomorphism_v2.tex date: May 7, 2018 
design by adding $z=4 k$ rows for which the inner products of the column pairs equal $-2,0,2,-2,0$ and -2 , respectively.

In the extra $4 k$ rows, the second column should add up to -2 . Therefore, $a=2 k-1$ and $b=2 k+1$. The third column's inner products with the first and the second column should equal 0 and 2 , respectively. Therefore, $c=k-1, d=k, e=k+1$ and $f=k$. For the constants $p, q, r, s, t, u, v$ and $w$, we can derive four equations based on the values of $c, d, e$ and $f$ and three equations based on the inner products with column 1-3 necessary to make the conference design orthogonal. These equations are as follows.

$$
\begin{aligned}
p+q & =k-1 \\
r+s & =k \\
t+u & =k+1 \\
v+w & =k \\
p-q+r-s+t-u+v-w & =2 \\
p-q+r-s-t+u-v+w & =0 \\
p-q-r+s+t-u-v+w & =-2
\end{aligned}
$$

We want to establish that $r \geq 1, t \geq 1, u \geq 1$ and $v \geq 1$. We proof that $r \geq 1$; proofs for the remaining three inequalities are similar.

Suppose that $r=0$. In that case, adding up Equations (3) and (4) results in

$$
2(p-q)-2 s=2 \text {. }
$$

By Equation (2), $s=k$. Inserting this value in Equation 5 results in $p-q=$ $k+1$. Combining with Equation (1) results in $p=k$ and $q=-1$. This

TABLE 12

General shape of the nonzero parts of four-column conference designs

\begin{tabular}{rrrr}
\hline$x_{1}$ & $x_{2}$ & $x_{3}$ & $x_{4}$ \\
\hline $\mathbf{1}_{z}$ & $\mathbf{1}_{a}$ & $\mathbf{1}_{c}$ & $\mathbf{1}_{p}$ \\
& & & $-\mathbf{1}_{q}$ \\
& & $-\mathbf{1}_{d}$ & $\mathbf{1}_{r}$ \\
& & & $-\mathbf{1}_{s}$ \\
& $-\mathbf{1}_{b}$ & $\mathbf{1}_{e}$ & $\mathbf{1}_{t}$ \\
& & & $-\mathbf{1}_{u}$ \\
& & $-\mathbf{1}_{f}$ & $\mathbf{1}_{v}$ \\
& & & $-\mathbf{1}_{w}$ \\
\hline
\end{tabular}

imsart-aos ver. 2014/10/16 file: AoS-DSDisomorphism_v2.tex date: May 7, 2018 
is a contradiction, because the length of the vectors in Table 12 cannot be negative. We conclude that $r \geq 1$.

The fact that $r \geq 1, t \geq 1, u \geq 1$ and $v \geq 1$ shows that each $N \times 4$ conference design of series A must contain a row with $\left(x_{1}, x_{2}, x_{3}, x_{4}\right)=(1,1,-1,1)$ (by the condition on $r$ ), a row with $\left(x_{1}, x_{2}, x_{3}, x_{4}\right)=(1,-1,1,1)$ (by the condition on $t$ ), a row with $\left(x_{1}, x_{2}, x_{3}, x_{4}\right)=(1,-1,1,-1)$ (by the condition on $u$ ) and a row with $\left(x_{1}, x_{2}, x_{3}, x_{4}\right)=(1,-1,-1,1)$ (by the condition on $v$ ). Therefore, it is without loss of generality that the rows $5-8$ of the conference design can be taken to be those mentioned here. Any further run size then can only be obtained by taking a column of ones as the first column and inserting the columns of a three-factor orthogonal array as the second, third and fourth columns.

A.2. Series B. The initial four rows of a conference design in series B form a conference design by themselves. Therefore, in any further set of $4 k$ rows, the columns must be orthogonal. By convention, the first column's elements all equal +1 . The columns 2,3 and 4 must then form an orthogonal array.

A.3. Series C. For the initial four rows of an $N \times 4$ conference design in series C, the six inner products of the column pairs in Table 8 all equal 2 . The initial rows can be turned into a conference design by adding $z=4 k+2$ rows for which the inner products of the column pairs all equal -2 .

Referring to the general outline in Table 12, the second column in the extra $4 k+2$ rows should add up to -2 . Therefore, $a=2 k$ and $b=2 k+2$. The third column's inner products with the first and the second column should both equal -2 . Therefore, $c=k-1, d=k+1, e=k+1$ and $f=k+1$. For the constants $p, q, r, s, t, u, v$ and $w$, we can derive four equations based on the values of $c, d, e$ and $f$ and three equations based on the inner products with column 1-3 necessary to make the conference design orthogonal. These equations are as follows.

$$
\begin{aligned}
p+q & =k-1 \\
r+s & =k+1 \\
t+u & =k+1 \\
v+w & =k+1 \\
p-q+r-s+t-u+v-w & =-2 \\
p-q+r-s-t+u-v+w & =-2 \\
p-q-r+s+t-u-v+w & =-2
\end{aligned}
$$


We want to establish that $s \geq 2, u \geq 2$ and $v \geq 2$. We proof that $s \geq 2$; proofs for the remaining two inequalities are similar.

Adding up Equations (8) and (9) results in

$$
(p-q)+(r-s)=-2 .
$$

Suppose that $s=0$. In that case, by Equation (7), $r=k+1$. Inserting this value in Equation (10) results in $p-q=-k-3$. Combining with Equation (6) results in $p=-2$ and $q=k+1$. This is a contradiction, because the length of the vectors in Table 12 cannot be negative. We conclude that $s \geq 1$. It is easy to show by a similar reasoning that supposing $s=1$ would also result in a contradiction. We thus have proven that $s \geq 2$.

The fact that $s \geq 2, u \geq 2$ and $v \geq 2$ shows that each $N \times 4$ conference design of series $\mathrm{C}$ must contain two rows with $\left(x_{1}, x_{2}, x_{3}, x_{4}\right)=(1,1,-1,-1)$ (by the condition on $s$ ), two rows with $\left(x_{1}, x_{2}, x_{3}, x_{4}\right)=(1,-1,1,-1)$ (by the condition on $u$ ), and two rows with $\left(x_{1}, x_{2}, x_{3}, x_{4}\right)=(1,-1,-1,1)$ (by the condition on $v$ ). Therefore, it is without loss of generality that the rows 5-10 of the conference design can be taken to be those mentioned here. Any further run size then can only be obtained by taking a column of ones as the first column and inserting the columns of a three-factor orthogonal array as the second, third and fourth columns.

A.4. Series D. For the initial four rows of an $N \times 4$ conference design in series $\mathrm{D}$, the six inner products of the column pairs in Table 8 equal 2 (columns $x_{1}$ and $x_{2}$ ), 0 (columns $x_{1}$ and $x_{3}$ ), 0 (columns $x_{1}$ and $x_{4}$ ), 0 (columns $x_{2}$ and $x_{3}$ ), 0 (columns $x_{2}$ and $x_{4}$ ) and 2 (columns $x_{3}$ and $x_{4}$ ). The initial rows can be turned into a conference design by adding $z=4 k+2$ rows for which the inner products of the column pairs equal $-2,0,0,0,0$ and -2 , respectively.

Referring to the general outline in Table 12, the second column in the extra $4 k+2$ rows should add up to -2 . Therefore, $a=2 k$ and $b=2 k+2$. The third column's inner products with the first and the second column should both equal 0 . Therefore, $c=k, d=k, e=k+1$ and $f=k+1$. For the constants $p, q, r, s, t, u, v$ and $w$, we can derive four equations based on the values of $c, d, e$ and $f$ and three equations based on the inner products with column 1-3 necessary to make the conference design orthogonal. These equations are as follows.

imsart-aos ver. 2014/10/16 file: AoS-DSDisomorphism_v2.tex date: May 7, 2018 


$$
\begin{aligned}
p+q & =k \\
r+s & =k \\
t+u & =k+1 \\
v+w & =k+1 \\
p-q+r-s+t-u+v-w & =0 \\
p-q+r-s-t+u-v+w & =0 \\
p-q-r+s+t-u-v+w & =-2
\end{aligned}
$$

We want to establish that $u \geq 1$ and $v \geq 1$. We proof that $u \geq 1$; the proof for the remaining inequality is similar.

Adding up Equations (13) and (14) results in

$$
(p-q)+(t-u)=-1 .
$$

Suppose that $u=0$. In that case, by Equation (12), $t=k+1$. Inserting this value in Equation 15 results in $p-q=-k-2$. Combining with Equation (11) results in $p=-1$ and $q=k+1$. This is a contradiction, because the length of the vectors in Table 12 cannot be negative. We conclude that $u \geq 1$.

The fact that $u \geq 1$ and $v \geq 1$ shows that each $N \times 4$ conference design of series $\mathrm{D}$ must contain a row with $\left(x_{1}, x_{2}, x_{3}, x_{4}\right)=(1,-1,1,-1)$ (by the condition on $u$ ) and a row with $\left(x_{1}, x_{2}, x_{3}, x_{4}\right)=(1,-1,-1,1)$ (by the condition on $v$ ). Therefore, it is without loss of generality that the rows 5 and 6 of the conference design can be taken to be those mentioned here. Any further run size then can only be obtained by taking a column of ones as the first column and inserting the columns of a three-factor orthogonal array as the second, third and fourth columns. 


\section{APPENDIX B: BEST CONFERENCE MATRIX OF ORDER 24}

\begin{tabular}{cccccccccccccccccccccccccccccccc}
\hline 0 & + & + & + & + & + & + & + & + & + & + & + & + & + & + & + & + & + & + & + & + & + & + & + \\
+ & 0 & - & - & - & - & - & - & - & - & - & - & - & + & + & + & + & + & + & + & + & + & + & + \\
+ & + & 0 & - & - & - & - & - & + & + & + & + & + & - & - & - & - & - & - & + & + & + & + & + \\
+ & + & + & 0 & - & - & - & + & - & - & + & + & + & - & - & + & + & + & + & - & - & - & - & + \\
+ & + & + & + & 0 & - & + & - & - & + & - & - & + & - & + & - & - & + & + & - & - & + & + & - \\
+ & + & + & + & + & 0 & - & + & + & - & - & - & - & - & - & - & + & - & + & + & + & - & + & - \\
+ & + & + & + & - & + & 0 & - & + & + & - & - & - & + & - & + & - & + & - & - & + & - & - & + \\
+ & + & + & - & + & - & + & 0 & + & - & - & + & - & + & + & - & + & - & - & - & - & + & - & + \\
+ & + & - & + & + & - & - & - & 0 & + & + & - & + & + & + & + & + & - & - & + & - & - & - & - \\
+ & + & - & + & - & + & - & + & - & 0 & + & + & - & + & + & - & - & - & + & - & + & + & - & - \\
+ & + & - & - & + & + & + & + & - & - & 0 & - & + & - & + & + & - & - & - & - & + & - & + & + \\
+ & + & - & - & + & + & + & - & + & - & + & 0 & - & - & - & + & - & + & + & + & - & + & - & - \\
+ & + & - & - & - & + & + & + & - & + & - & + & 0 & + & - & - & + & + & - & + & - & - & + & - \\
+ & - & + & + & + & + & - & - & - & - & + & + & - & 0 & + & - & - & + & - & + & - & - & + & + \\
+ & - & + & + & - & + & + & - & - & - & - & + & + & - & 0 & + & + & - & - & + & + & + & - & - \\
+ & - & + & - & + & + & - & + & - & + & - & - & + & + & - & 0 & - & - & + & + & - & + & - & + \\
+ & - & + & - & + & - & + & - & - & + & + & + & - & + & - & + & 0 & - & + & - & + & - & + & - \\
+ & - & + & - & - & + & - & + & + & + & + & - & - & - & + & + & + & 0 & - & - & - & + & + & - \\
+ & - & + & - & - & - & + & + & + & - & + & - & + & + & + & - & - & + & 0 & + & + & - & - & - \\
+ & - & - & + & + & - & + & + & - & + & + & - & - & - & - & - & + & + & - & 0 & + & + & - & + \\
+ & - & - & + & + & - & - & + & + & - & - & + & + & + & - & + & - & + & - & - & 0 & + & + & - \\
+ & - & - & + & - & + & + & - & + & - & + & - & + & + & - & - & + & - & + & - & - & 0 & + & + \\
+ & - & - & + & - & - & + & + & + & + & - & + & - & - & + & + & - & - & + & + & - & - & 0 & + \\
+ & - & - & - & + & + & - & - & + & + & - & + & + & - & + & - & + & + & + & - & + & - & - & 0 \\
\hline
\end{tabular}

\section{SUPPLEMENTARY MATERIAL}

\section{Supplement A: Conference matrices of order $\mathbf{8 2}$}

(doi: COMPLETED BY THE TYPESETTER; .zip). We provide Matlab code to construct the 26 non-isomorphic conference matrices of order 82 of Hurkens and Seidel (1985) and to evaluate the $F_{4}$ vector of the definitive screening designs with 82 factors based on these matrices.

\section{REFERENCES}

Delsarte, P., Goethals, J. M. and Seidel, J. J. (1971). Orthogonal matrices with zero diagonal II. Canadian Journal of Mathematics 23 816-832.

Deng, L. Y. and TANG, B. (1999). Generalized resolution and minimum aberration criteria for Plackett-Burman and other nonregular factorial designs. Statistica Sinica 9 1071-1082.

Deng, L. Y. and TANG, B. (2002). Design selection and classification for Hadamard matrices using generalized minimum aberration criteria. Technometrics 44 173-184.

Dougherty, S., Simpson, J. R., Hill, R. R., Pignatiello, J. J. and White, E. D. (2015). Effect of Heredity and Sparsity on Second-Order Screening Design Performance. Quality and Reliability Engineering International 31 355-368. 
Fidaleo, M., Lavecchia, R., Petrucci, E. and Zuorro, A. (2016). Application of a novel definitive screening design to decolorization of an azo dye on boron-doped diamond electrodes. International Journal of Environmental Science and Technology 13 835-842.

Georgiou, S. D., Stylianou, S. and Aggarwal, M. (2014). Efficient three-level screening designs using weighing matrices. Statistics 48 815-833.

Greig, M., HaAnPÄ̈̈, H. and KASKI, P. (2006). On the coexistence of conference matrices and near resolvable $2-(2 k+1, k, k 1)$ designs. Journal of Combinatorial Theory, Series A 113 703-711.

Hurkens, C. A. J. and Seidel, J. J. (1985). Conference Matrices from Projective Planes of Order 9. European Journal of Combinatorics 6 49-57.

Jones, B. and Nachtsheim, C. J. (2011). A class of three-level designs for definitive screening in the presence of second-order effects. Journal of Quality Technology 43 $1-15$.

Jones, B. and Nachtsheim, C. J. (2013). Definitive screening designs with added twolevel categorical factors. Journal of Quality Technology 45 121-129.

Jones, B. and Nachtsheim, C. J. (2017). Effective design-based model selection for definitive screening designs. Technometrics 59 319-329.

Mee, R. W., Schoen, E. D. and Edwards, D. E. (2017). Selecting an Orthogonal or Non-Orthogonal Two-Level Design for Screening. Technometrics 59 305-318.

Nachtsheim, A. C., Shen, W. and Lin, D. K. J. (2017). Two-level augmented definitve screening designs. Journal of Quality Technology 49 93-107.

Nguren, N. K. and Pham, T. D. (2016). Small Mixed-Level Screening Designs with Orthogonal Quadratic Effects. Journal of Quality Technology 48 405-414.

PAtil, M. V. (2017). Multi response simulation and optimization of gas tungsten arc welding. Applied Mathematical Modelling 42540 - 553.

Schoen, E. D. (2018). Supplement to "A Classification Criterion for Definitive Screening Designs". DOI.

Schoen, E. D., Vo-Thanh, N. and Goos, P. (2017). Two-Level Orthogonal Screening Designs With 24, 28, 32, and 36 Runs. Journal of the American Statistical Association 112 1354-1369.

XiAO, L., Lin, D. K. and BAI, F. (2012). Constructing definitive screening designs using conference matrices. Journal of Quality Technology 44 1-7.

Faculty of Bioscience Engineering

Kasteelpark Arenberg 30 - Box 2456

3001 LEUVEN

BELGIUM

E-MAIL: eric.schoen@kuleuven.be peter.goos@kuleuven.be
Dept. of Engineering Management PRINSSTRAAT 13 2000 AntwERPEN BELGIUM

E-MAIL: pieter.eendebak@gmail.com 